\title{
PARABOLIC ANISOTROPIC PROBLEMS WITH LOWER ORDER TERMS AND INTEGRABLE DATA
}

\author{
Moussa Chrif, Said El Manouni And Hassane Huiaj
}

Abstract. In this paper we are concerned with the study of a class of second-order quasilinear parabolic equations involving Leray-Lions type operators with anisotropic growth conditions. By an approximation argument, we estabilsh the existence of entropy solutions in the framework of anisotropic parabolic Sobolev spaces when the initial condition and the data are assumed to be merely integrable. In addition, we prove that entropy solutions coincide with the renormalized solutions.

Mathematics subject classification (2010): 35K55, 35K58, 35K59, 46E35.

Keywords and phrases: Quasilinear anisotropic parabolic equations, anisotropic Sobolev spaces, penalization methods, entropy solutions, renormalized solutions.

\section{REFERENCES}

[1] M. H. Abdou, M. Chrif, S. El Manouni and H. Huiaj, On a class of nonlinear anisotropic parabolic problems, Proc. Roy. Soc. Edinburgh Sect. A 146 (2016), no. 1, 1-21.

[2] F. Andreu, J. M. Mazón, S. Segura de León, ANd J. Toledo, Existence and uniqueness for a degenerate parabolic equation with L1-data, Trans. Amer. Math. Soc. (1999), 351 (1), 285-306.

[3] S. Antontsev AND M. Chipot, Anisotropic equations: uniqueness and existence results, Differential and Integral Equations, 21 (2008), 401-419.

[4] A. Alberico, G. Di Blasio AND F. Feo, Comparison results for nonlinear anisotropic parabolic problems, Atti Accad. Naz. Lincei Rend. Lincei Mat. Appl. 28 (2017), 305-322.

[5] P. Bénilan, L. Boccardo, T. Gallouët, R. Gariepy, M. Pierre and J. L. Vázquez, An L1-theory of existence and uniqueness of solutions of nonlinear elliptic equations, Ann. Scuola Norm. Sup. Pisa Cl. Sci. 4, (1995), 241-273.

[6] D. Blanchard And F. MURat, Renormalized solutions of nonlinear parabolic problems with $L^{1}$ data, Existence and uniqueness, Proc. Roy.Soc. Edinburgh Sect. A 127, (1997), 1137-1152.

[7] D. Blanchard, F. Murat and H. Redwane, Existence and Uniqueness of a Renormalized Solution for a Fairly General Class of Nonlinear Parabolic Problems, J. Differential Equations. Vol 177, (2001), 331-374.

[8] M. Bendahmane, M. Chrif And S. El Manouni, An approximation result in generalized anisotropic Sobolev spaces and applications, Z. Anal. Anwend. 30 (2011), no. 3, 341-353.

[9] M. Bendahmane, P. Wittbold And A. Zimmermann, Renormalized solutions for a nonlinear parabolic equation with variable exponents and $L^{1}$-data, J. Differential Equations 249 (2010), no. 6, $1483-1515$.

[10] M. Bendahmane AND K. H. KARlSEn, Renormalized solutions of an anisotropic reactiondiffusion, Comm. Pure Appl. Anal. 5 (4) (2006), 733-762.

[11] M. Bendahmane AND K. H. Karlsen, Nonlinear anisotropic elliptic and parabolic equations in $\mathbb{R}^{N}$ with advection and lower order terms and locally integrable data, Potential Analysis, (2005), vol. 22, 207-227.

[12] M. Bendahmane And S. MaZen, Entropy Solution for Anisotropic Reaction-Diffusion-Advection Systems with $L^{1}$ Data, Rev. Mat. Complut. (2005), 18, No. 1, 49-67. 
[13] L. Boccardo, J. I. Diaz, D. Giachetti, And F. Murat, Existence of a solution for a weaker form of a nonlinear elliptic equation. In Recent advances in nonlinear elliptic and parabolic problems, (Nancy, 1988), volume 208 of Pitman Res. Notes Math. Ser., pages 229-246. Longman Sci. Tech., Harlow, 1989.

[14] L. Boccardo, A. Dall' Aglio, T. Gallouët and L. Orsina, Nonlinear parabolic equations with measure data, J. Funct. Anal. 147 (1997), no. 1, 237-258.

[15] L. Boccardo, T. GallouËT AND P. MarCellini, Anisotropic equations in $L^{1}$, Differential Integral Equations 9 (1996), no. 1, 209-212.

[16] L. Boccardo, T. GAllouët ANd J. L. VÁzQuez, Some regularity results for some nonlinear parabolic equations in $L^{1}$, Rend. Sem. Mat. Univ. Special Issue (1991), 69-74.

[17] M. Chrif And S. El Manouni, On a strongly anisotropic equation with $L^{1}$ data, Appl. Anal. 87 (7) (2008), 865-871.

[18] M. Chrif, S. El Manouni And F. Mokhtari, Strongly anisotropic elliptic problems with regular and $L^{1}$ data, Portugal. Math. 72 (4) (2015), 357-391.

[19] A. Di CAstro, Anisotropic elliptic problems with natural growth terms, Manuscripta Math. 135 (3-4) (2011), 521-543.

[20] R. J. DiPerna AND P.-L. Lions, On the Cauchy problem for Boltzmann equations: global existence and weak stability, Ann. of Math. (2), (1989) vol. 130 (2):321-366.

[21] T. Donaldson, Inhomogeneous Orlicz-Sobolev spaces and nonlinear parabolic initial boundary value problems, J. Differential Equations 16 (1974), 201-256.

[22] A. Elmahi and D. Meskine, Parabolic equations in Orlicz, spaces, J. London Math. Soc. (2) 72 (2005), 410-428.

[23] I. Fragalá, F. Gazzola And B. KaWohl, Existence and nonexistence results for anisotropic quasilinear elliptic equations, Annales de l'I.H.P. Analyse non linéaire, Volume 21 (2004), no. 5, 715-734.

[24] E. Hewitt and K. Stromberg, Real and abstract analysis, Springer-Verlag, Berlin Heidelberg New York, 1965.

[25] R. LANDES AND V. Mustonen, A strongly nonlinear parabolic initial-boundary value problems, Ark. Mat. 25 (1987), 29-40.

[26] R. LANDES AND V. Mustonen, On parabolic initial-boundary value problems with critical growth for the gradient, Ann. Inst. H. Poincaré, 11 (2) (1994), 135-158.

[27] F. LI, Anisotropic elliptic equations in $L^{m}$, J. Convex Anal. 8 (2) (2001), 417-422.

[28] J. L. Lions, Quelque Méthodes de Résolution des Problémes aux Limites Non Linéaires (in French), Paris: Dunod; Gauthier-Villars 1969.

[29] P.-L. Lions, Mathematical topics in fluid mechanics, Vol. 1, volume 3 of Oxford Lecture Series in Mathematics and its Applications. Oxford University Press, New York, 1996.

[30] M. MiHĂILESCU, V. RĂDULESCU, Existence and multiplicity of solutions for quasilinear nonhomogeneous problems: An Orlicz-Sobolev space setting, J. Math. Anal. Appl. 330 (2007), 416-432.

[31] M. MinĂILESCU, P. PUCCI AND V. RĂDULESCU, Eigenvalue problems for anisotropic quasilinear elliptic equations with variable exponent, J. Math. Anal. Appl. 340 (2008), 687-698.

[32] F. Mokhtari, Anisotropic parabolic problems with measures data, Differ. Equ. Appl. 2 (2010), 123150.

[33] J. RÁKosníK, Some remarks to anisotropic Sobolev spaces I, Beiträge Anal. 13 (1979), 55-68.

[34] J. RÁKosník, Some remarks to anisotropic Sobolev spaces II, Beiträge Anal. 15 (1981), 127-140.

[35] M. RuZICKA, Electrorheological fluids: modeling and mathematical theory, Springer-Verlag, Berlin, 2002.

[36] J. Simon, Compact sets in the space $L^{p}(0, T ; B)$, Ann. Mat. Pura Appl. (4) 146 (1987), 65-96. 